\title{
Neutralization of HMGB1 improves fracture healing and $\gamma \delta$ T lymphocyte counts at the fracture site in a polytrauma rat model
}

\author{
Preeti J. Muire*, Joshua J. Avila, Alicia L. Lofgren and Joseph C. Wenke
}

\begin{abstract}
Purpose: Delayed fracture healing is a common consequence of polytrauma (PT) occurring in patients with multiple injuries. We believe that when early release of high mobility group box 1 (HMGB1) molecules from necrotic tissues exceed their normal levels in blood, they dysregulate immune responses associated with normal healing. This study investigates the detrimental effect of such dysregulate immune responses by targeting HMGB1 in a PT rat model with debilitating injuries. We hypothesized that neutralization of extracellular HMGB1 immediately post-trauma would ameliorate local immune dysregulation and improve fracture healing in a PT rat model.

Methods: PT rats received a single dose of either anti-rat HMGB1 polyclonal antibody (PT-Ab HMGB1) or IgY isotype (PT-IgY), were left untreated (PT-C), or had a single injury/osteotomy only (OST). Fracture healing was evaluated by micro-computed tomography $(\mu \mathrm{CT})$ and histology at 5 weeks; and macrophages and T cell counts within the fracture site were determined with flow cytometry at 1 week.

Results: Notably, bone regeneration within the fracture site in PT-Ab HMGB1 rats was improved with comparable connective tissue organization than PT-C rats. Further, only $\gamma \delta T C R^{+} T$ cells, but not macrophages and CD4 ${ }^{+}$and CD $8^{+}$ T cells, were diminished at the fracture site in PT-C and PT-IgY rats. Interestingly, the PT-Ab HMGB1 rats had increased $\gamma \delta T C R^{+} T$ cells compared to PT-C and PT-IgY, suggesting their potential role in regulating fracture healing.

Conclusions: Therefore, the initial burst of systemic HMGB1 following trauma may have a role in regulating bone regeneration via the modulation of a subclass of T cells within the fracture site, suggesting it's importance as a therapeutic target in PT to combat immune dysregulation and delayed fracture healing.
\end{abstract}

Keywords: Delayed fracture healing, Severe trauma, Osteoimmunology, DAMPs

\section{Introduction}

Fracture healing is an intricate process, and a well-balanced immune response is integral to bone repair and remodeling of complicated and straightforward fractures $[1,2]$. Polytrauma (PT) injuries sustained on the battlefield are associated with extensive tissue necrosis, elevated damage associated molecular patterns (DAMPs), immune dysregulation, and delayed fracture healing or

*Correspondence: preetimuire@gmail.com

Combat Wound Care Department, US Army Institute of Surgical Research, JBSA Fort Sam, Houston, TX 78234, USA nonunion depending on the injury severity [3]. Presently, with the current standard of care there does not appear to be a discernable improvement in fracture healing in combat casualties and it remains the highest priority in military research.

High mobility group box protein 1 (HMGB1) is a potent DAMP that mounts early systemic and local inflammation following polytraumatic injuries [4-6]. When present at elevated levels, HMGB1 activates pathways that potentiate further tissue necrosis that further increases HMGB1 levels in a positive feedback mechanism, thereby causing detrimental outcomes 
such as cellular exhaustion and impaired healing [6, 7]. Increased extracellular HMGB1 expression has been reported in several sterile injury models, including collagen-induced arthritis or during the spontaneous development of arthritis in mice [8]. Systemic administration of neutralizing HMGB1 antibody significantly ameliorated these autoimmune diseases, indicated by reduced weight loss and diminished cartilage and bone destruction in arthritic joints $[8,9]$. Another study highlighted that HMGB1 induced osteoclastogenic bone destruction associated with oral squamous cancer, whereas blocking of the HMGB1:RAGE/TLR4 axis inhibited bone destruction in a mouse model [10]. Despite the well-documented detrimental role of HMGB1 as a mediator of normal remodeling and inflammatory bone loss in simple fractures [11, 12], the role of HMGB1 in the context of PT fractures has not been elucidated. We suppose that while HMGB1 may have a role in regulating fracture repair, it may function in a dose-dependent manner. Thereby we postulate that targeting the initial burst of HMGB1 during the early minutes to hours post-injuries may be a promising approach to regulate its systemic levels throughout fracture healing.

HMGB1 induces lymphocyte activation and the cytokines secreted by lymphocytes harboring within the fracture microenvironment affect bone metabolism $[13,14]$. Previous studies that elucidated the role of the adaptive immune system in fracture healing found that the lack of lymphocytes led to accelerated bone mineralization with overall deteriorated bone quality in $\mathrm{RAG1}^{-l-}$ knockout mice, suggesting a regulatory role of lymphocytes in fracture repair [15-17]. Further, the osteogenic role of $\mathrm{T}$ cells was elucidated in the context of extracellular matrix and collagen deposition in fractures [18]. Among the $\mathrm{CD}^{+}$lymphocytes, the $\mathrm{CD}^{+} \mathrm{T}$ cells are thought to have an osteogenic role via the activation of mesenchymal stem cells (MSCs) [19]. Moreover, emerging data indicate that innate lymphocytes, particularly the $\gamma \delta \mathrm{T}$ cells, may also be necessary for bone regeneration [20]. $\gamma \delta \mathrm{T}$ cells are early responders and can become activated during the acute phase post-trauma in response to DAMPS, thereby facilitating inflammation, chemotaxis, and cell lysis via the production of cytokine and growth factors [21]. This study investigates the detrimental effect of dysregulated immune responses mounted by HMGB1 in a PT rat model, with a focus on fracture repair and early cellular dynamics of macrophages and $\mathrm{T}$ cells at the fracture site. We hypothesized that neutralization of extracellular HMGB1 with a single dose of anti-HMGB1 antibody immediately post-trauma would ameliorate immune dysregulation and improve fracture repair in a PT rat model.

\section{Materials and methods}

\section{Animals and surgical care}

Male Sprague-Dawley (SD) rats aged $14.2 \pm 1.2$ weeks with the average weight of $372.5 \mathrm{~g} \pm 19.2 \mathrm{~g}$ were used in this study. All rats were housed in a specific pathogenfree facility, provided with unlimited access to food and water, and unrestricted activity before and after all procedures. Research was conducted in compliance with the Animal Welfare Act, the implementing Animal Welfare regulations, and the principles of the Guide for the Care and Use of Laboratory Animals, National Research Council. The Institutional Animal Care \& Use Committee (IACUC) at the United States Army Institute of Surgical Research approved all research conducted in this study. IACUC approval number is A-16-044 and date is June $28^{\text {th }}, 2016$. The facility where this research was conducted is fully accredited by the AAALAC. A pre-surgical dose of Buprenorphine SR-LAB (1.2 mg/ $\mathrm{kg}$, subcutaneously) was administered for pain management at least $15 \mathrm{~min}$ before surgery. At surgery, rats were anesthetized and maintained with 1-3\% isoflurane and oxygen delivered via a nose cone on a Bain circuit connected to the rodent gas anesthesia machine (VetEquip Inc., Pleasanton, Ca). Naïve rats were not surgically manipulated and served as baseline controls. Post-surgery, rats did not receive any prophylactic antibiotics but were assessed closely for signs of distress and body weight. Rats with $\geq 10 \%$ of body weight loss relative to pre-surgery weight received $3 \mathrm{ml}$ sterile surgical saline once daily subcutaneously. At the designated endpoints, i.e., 1 or 5 weeks, rats were anesthetized and humanely euthanized by cardiac exsanguination or an intracardiac (IC) overdose of sodium pentobarbital. Following euthanasia, the tissues were harvested for downstream processing. This study was carried out in compliance with the ARRIVE guidelines [22].

\section{Surgery}

Rats were divided into two cohorts of trauma representing single $3 \mathrm{~mm}$ femoral osteotomy (OST)/normal and polytrauma (PT)/delayed fracture repair models. PT rats underwent three traumas, i.e., a $3 \mathrm{~mm}$ femoral osteotomy, a blunt chest contusion with a $0.3 \mathrm{~kg}$ weight dropped from a height of $68 \mathrm{~cm}$ to exert $\sim 2 \mathrm{~J}$ of energy on the rat's chest, and a $100{ }^{\circ} \mathrm{C}$ water scald burn for $10 \mathrm{~s}$ of approximately $20 \%$ of the total body surface area. All trauma and surgical procedures were previously described in detail and can be found in [23].

Anti-HMGB1 antibody and IgY isotype antibody injections Immediately post trauma procedures, sub-cohorts of PT rats designated as PT-Ab HMGB1, PT-Ab HMGB1 $3 \times$ and PT-IgY, respectively, received either a single dose 
(1 dose/day 0) or three consecutive doses (1dose/day 0, 1, and 2) of chicken anti-HMGB1 neutralizing polyclonal antibody or isotype control chicken IgY antibody (Shinotest, Tokyo, Japan; $2 \mathrm{mg} / \mathrm{kg}$ per dose, intraperitoneal (IP)). PT rats which did not receive antibody injection were designated as PT-C. All rats were recovered in clean cages with continued monitoring.

\section{Blood collection and processing for ELISA}

Blood was collected at 1 week post-trauma (wpt) from anesthetized rats $(n=4-5 / \mathrm{OST}, \mathrm{PT}-\mathrm{C}, \mathrm{PT}-\mathrm{IgY}$ and PT-Ab HMGB-1) via tail vein cannulation in pre-weighed EDTA tubes and centrifuged at $1000 \mathrm{xg}$ for $10 \mathrm{~min}$ to separate the plasma for HMGB1 protein quantification. Plasma was stored at $-80^{\circ} \mathrm{C}$ until used for the HMGB1 ELISA kit (IBL International), per manufacturer's instructions.

\section{Micro-computed tomography $(\mu \mathrm{CT})$}

At 5 wpt, OST, PT-C, and PT-Ab HMGB1 rats $(n=10-11)$ the right femur was harvested without disturbing the callus, an ex vivo radiograph was acquired (Faxitron UltraFocus), and was then fixed in 10\% neutral buffered formalin followed by $\mu \mathrm{CT}$ scans (VivaCT40, SCANCO Medical). The osteotomy site was scanned at high resolution with a $10.5 \mu \mathrm{m}$ voxel size at a voltage of $70 \mathrm{kVp}$, a current of $114 \mu \mathrm{A}$, and an integration time of $300 \mathrm{~ms}$. Images were converted to 8-bit bitmap files using Image J (National Institutes of Health, Bethesda, $\mathrm{MA}$ ) and reoriented with DataViewer (Bruker-MicroCT, Kontich, Belgium) so that the slices were in the plane of the defect border. Regions of interest (ROI) and three-dimensional analysis were completed using CTan (Bruker-MicroCT, Kontich, Belgium). The volume of interest (VOI) extended $\sim 2.6 \mathrm{~mm}$ (251 slices) and was centered within the osteotomy. For the values entitled 'Defect,' a polygonal ROI was drawn around the portion in which an intact cortex was first seen, proximal and distal to the defect, and interpolated throughout the slices to simulate the area in which the intact femur should be present. For the values entitled 'Continuous Callus', an ROI was drawn tightly around any new callus formation that extended from the cortex (any islands of bone not connected to the cortex were excluded). A new ROI was drawn every 25 slices, or more as needed, based on the rapidity of change in callus shape, and interpolated. A global threshold was calculated using the Otsu method [24], and a value of 107 was determined to be the delineation between non-mineralized and mineralized tissue. The three-dimensional image reconstructions were used to quantify bone. Values are reported as either bone volume fraction, which is the ratio between bone volume and total volume within the given $\mathrm{VOI}(\mathrm{BV} / \mathrm{TV}, \%)$, or volume of bone $\left(\mathrm{mm}^{3}\right)$. These measurements were calculated in the three cohorts, as previously described [23].

\section{Histology of femoral defect}

Femurs ( $n=4-8$ /OST, PT-C, and PT-Ab HMGB1) were harvested and processed for histological analysis. Briefly, femurs were fixed in 10\% neutral buffered formalin, rinsed in type 1 ultra-pure water, decalcified in formic acid for 21 days and embedded in paraffin. Longitudinal sections of $8 \mu \mathrm{m}$ thickness were stained with hematoxylin and eosin (H\&E) or Masson trichrome (MT), and estimation of bone formation was obtained by bright field microscopy with an Olympus BX41 microscope and images were captured using CellSens standard software (Olympus). The scoring key used to assess callus quality is as follows: $0-$ Bony union; 1 - Callus is composed primarily of new bone ( $>50 \%) ; 2-$ Callus is composed of fibrous tissue $(>50 \%)$, new bone $(<50 \%)$, skeletal muscle and/or adipose tissue; $3-$ Callus is composed of fibrous tissue $(<50 \%)$, new bone $(<50 \%)$, skeletal muscle and/ or adipose tissue; 4-Callus is composed of acute hemorrhage, spindle cells and inflammatory cells (new bone present); and 5-Callus is composed of acute hemorrhage, spindle cells and inflammatory cells (no new bone). The scoring key used to assess the extent of hemosiderin-laden macrophages present at the fracture site is as follows: 0 - Absent; 1-Acute hemorrhage only; 2Chronic hemorrhage only (with hemosiderin-laden macrophages); and 3-Acute and chronic hemorrhage. The scoring key used to assess the presence of osteoblasts and osteoclasts in the bone samples is as follows: $0-$ None; 1 - Minimal; 2 - Mild; 3 - Moderate; 4 - Marked; and 5 - Severe, similar to previously published [23]. All slides were reviewed and scored by a board-certified veterinary pathologist evaluating the callus quality, the presence of hemosiderin-laden macrophages, osteoblasts and osteoclasts, cellular composition and collagen organization.

\section{Flow cytometry}

At $1 \mathrm{wpt}$, the fracture hematoma or soft callus was collected from the osteotomised femur $(n=4-5 / \mathrm{OST}$, PT-C, PT-IgY, and PT-Ab HMGB1) accompanied by a rinse of the area surrounding the defect with $10 \mathrm{ml}$ of sterile PBS. A single-cell suspension was prepared for flow cytometry. The fracture site's cell collection process was standardized across all the animal groups in this study except for the naïve rats whose whole bone marrow cells were collected from the uninjured femur. Fracture site specimens were passed through a $40 \mu \mathrm{m}$ filter to prepare a single-cell suspension. Cells were washed in FACS buffer and RBCs were lysed. Cells were resuspended in FACS buffer and counted by trypan blue exclusion method using the automated cell counter (Countess, Invitrogen), and $1 \times 10^{6}$ 
Table 1 Antibodies and their titrated dilutions used for flow cytometry

\begin{tabular}{lll}
\hline Antibodies & Vendors & $\begin{array}{l}\text { Dilution } \\
\text { per } \\
\text { sample }\end{array}$ \\
\hline Anti-rat CD32/Fc block antibody & BD Bioscience & $1: 50$ \\
Anti-rat CD3 antibody & Miltenyi Biotech & $1: 50$ \\
Anti-rat CD4 antibody & Miltenyi Biotech & $1: 10$ \\
Anti-rat CD8a antibody & Miltenyi Biotech & $1: 10$ \\
Anti-rat y8TCR antibody & BioLegend & $1: 50$ \\
Anti-rat CD45 antibody & BD Biosciences & $1: 80$ \\
Anti-rat CD68 antibody & Miltenyi Biotech & $1: 10$ \\
Anti-rat CD86 antibody & Miltenyi Biotech & $1: 10$ \\
Anti-CD163 antibody & Novus Biologicals & $1: 150$ \\
Zombie violet & BioLegend & $1: 2000$ \\
\hline
\end{tabular}

cells/sample were stained. All wash steps were performed at $500 \mathrm{xg}$ for $3 \mathrm{~min}$ at $4{ }^{\circ} \mathrm{C}$. Cells were stained with a live/ dead stain, zombie violet dye (BioLegend), for $10 \mathrm{~min}$ at $4{ }^{\circ} \mathrm{C}$ and washed with PBS. The cells' Fc regions were then blocked with an anti-rat CD32/Fc block antibody before staining with the respective antibodies (Table 1). Cells were fixed with fixation buffer (R\&D systems) and washed twice with FACS buffer before proceeding with permeabilization step and intracellular staining. Data was acquired on a MACS Quant Analyzer 10 (Miltenyi Biotech). All antibodies were titrated before application. Appropriate isotype control antibodies, fluorescence minus one (FMOs), and single stained cells were used as controls for appropriate gating strategies. Compensation was performed with either single stained cells or beads to ensure there was no spillover within channels. Data were analyzed using Flow logic software (Miltenyi Biotech).

\section{Statistical analysis}

Statistical analyses were performed using GraphPad Prism version 8.0.0 for Windows, GraphPad Software, San Diego, California USA. Data were assessed for normality using the Q-Q plot, homoscedasticity plot, residual plot, D'Agostino and Pearson test, Shapiro-Wilk test, and the difference between means and median. ELISA data was assessed by Kruskal-Wallis test followed by Dunn's multiple comparison test. Bone regeneration within segmental defect of OST, PT-C and PT-Ab HMGB1 cohorts was assessed by ordinary one-way ANOVA with Dunnett's multiple comparison test for normally distributed data or Kruskal-Wallis test with Dunn's multiple comparison test if the data was not normally distributed. Flow cytometry data from blood was analyzed for statistical significance by performing a one-way ANOVA and Tukey's Post-hoc analysis at $a<0.05$ level of significance. Statistical significance was set at $p<0.05$.

\section{Results}

ELISA to validate neutralization of extracellular HMGB-1 in circulation

HMGB1 protein levels were quantified by ELISA in plasma from OST, PT-C, and PT-Ab HMGB1 rats at 1 wpt. Increased HMGB1 levels were observed at $1 \mathrm{wpt}$ in PT-C vs. OST and, statistical significance was noted in PT-C vs. PT-Ab HMGB1 $(p=0.03)$ (Fig. 1).

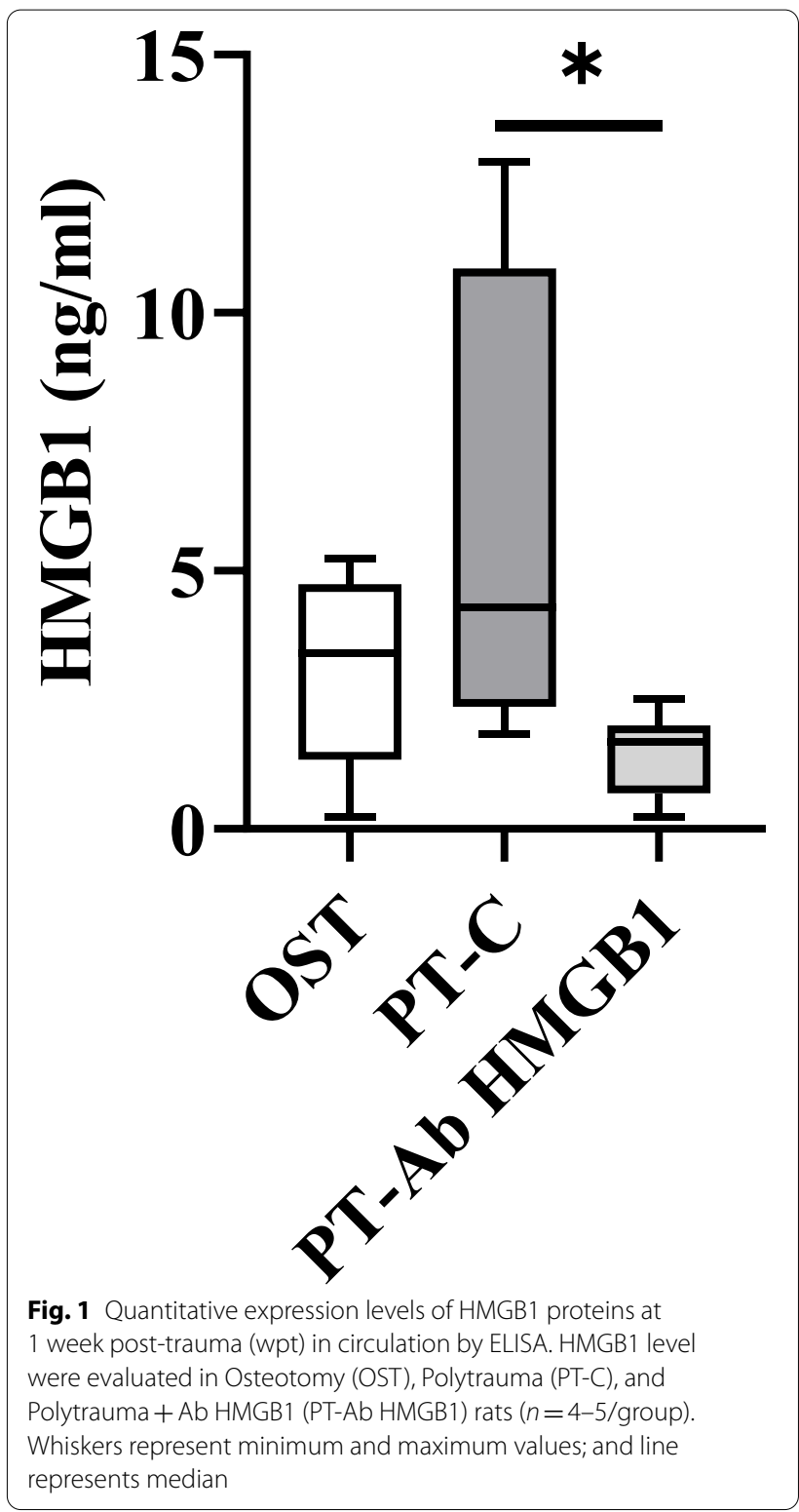


Micro-computed tomography $(\mu \mathrm{CT})$ to evaluate bone regeneration

To determine the role of HMGB1 in the regenerative process after PT, we assessed bone regeneration by evaluating bone healing with radiographic imaging as shown in Fig. 2A, and next measuring the amount of new bone

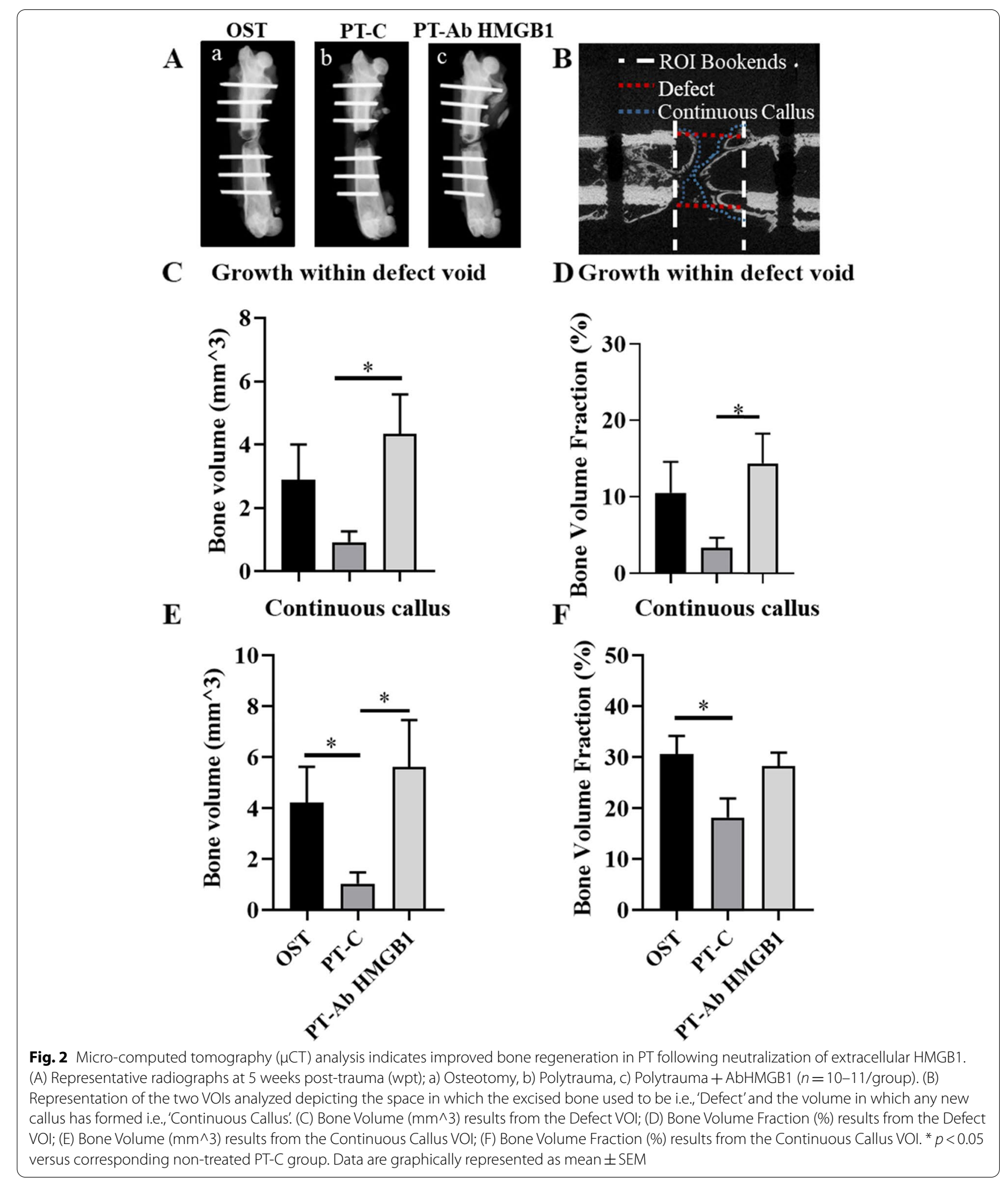


formed in the fracture site with $\mu \mathrm{CT}$ at $5 \mathrm{wpt}$ (Fig. 2B-F). Using the defect and continuous callus VOIs as described in the methods section, both the amount of mineralized tissue present $(\mathrm{BV})$ and the ratio of mineralized tissue to the total volume of the VOI (BV/TV) were compared across the three cohorts, i.e., OST, PT-C, and PT-Ab HMGB1/ PT-Ab HMGB1 3x. In a pilot study, PT rats received either a single dose (PT-Ab HMGB1) or three doses (1dose/day) (PT-Ab HMGB1 3x) of anti-HMGB1 antibody. Results suggest that PT-Ab HMGB1 rats had a mean threefold increase in bone growth compared to PT-C (Fig. 2D). Whereas, the PT-HMGB1 $3 \times$ rats had a mean onefold increase in bone growth (Supplementary Figure S1). For this reason, we proceeded with the single dose treatment for the entire study. Within the defect VOI, the bone volume and bone volume fraction in PT-Ab HMGB1 rats increased significantly compared to PT-C rats $(p=0.006$ and $p=0.01$, respectively). Further, within the continuous callus VOI, the PT-C average bone volume was significantly decreased compared with both OST $(p=0.02)$ and PT-Ab HMGB1 $(p=0.009)$. In comparison, the bone volume fraction was only different between OST and PT-C $(p=0.02)$ but not PT-Ab HMGB1 $(p=0.06)$. In both VOIs, there were no significant differences between PT-Ab HMGB1 and OST (Fig. 2C-F).

\section{Histological evaluation to confirm bone regeneration}

New bone growth was histologically evaluated using H\&E stain at 5 wpt to score the bone/callus formation and evaluate cellular composition within the defect of OST, PT-C, and PT-Ab HMGB1 rats. The scoring key used to assess callus quality is mentioned in the materials and methods section. Histological evaluation validated enhanced bone regeneration in OST and PT-Ab HMGB1 compared to PT-C (Fig. 3A-G). The loose connective tissue rich in adipocytes in PT-C (Fig. 3D and E) was comparable with the more mature, densely cellular, fibrous connective tissue in OST (Fig. 3B). An increase in hemosiderin-laden macrophages were reported to be observed as focal accumulations at sites of old hematomas in diseased conditions signifying trauma-induced hemorrhage
[25]. Hemorrhage can be acute (erythrocytes) or chronic (hemosiderin-laden macrophages). Acute hemorrhage occurs as soon as a vessel has ruptured. Hemosiderin is a break down product of heme and macrophages engulf the heme to remove it from the tissue. In severe trauma, the breakdown of erythrocytes takes a long time, during which hemosiderin-laden macrophages are present, and is characterized as chronic [26]. Chronic hemorrhage characterized by the presence of hemosiderin-laden macrophages was observed within the fracture defect of PT-C compared to OST and PT-AbHMGB1 (Fig. 3E and $\mathrm{H}$ ). Although the osteoclasts and osteoblasts were slightly less in PT-C compared to OST and PT-AbHMGB1, there were no statistically significant differences at 5 wpt (Fig. 3I and J).

\section{Qualitative assessment of collagen content in the defect}

Originating from mesenchymal stem cells, the osteoblasts produce the organic components of the bone extracellular matrix, i.e., type I collagen fibers that, along with cells, make up the connective tissue. Masson's Trichrome stain was used to evaluate organized collagen, which is seen by a deep saturation, to determine the connective tissue's quality, indicating mature connective tissue, which further suggests normal bone regeneration. In contrast, light saturation and sparse cells indicate immature connective tissue and poor bone regeneration. We noticed that at $5 \mathrm{wpt}$, in OST rats, the callus consists of new bone growth and fibrous connective tissue (Figs. 4A and B). In Fig. 4A, compare the immature fibrous connective tissue in the callus (400x) to the inset showing a mature connective tissue surrounding an artery. The connective tissue in the callus is moderately stained blue with partly organized collagen fibers compared to the mature connective tissue, stained deep blue, with densely packed organized collagen fibers (Fig. 4B). In the PT-C rats, the callus consists of lesser new bone growth and more immature fibrous connective tissue than the femur in the OST group (Figs. $4 \mathrm{C}$ and D). In Fig. 4C, compare the immature fibrous connective tissue in the callus (400x) to the inset showing a mature connective tissue of a tendon attached to the femur. The connective tissue in

\footnotetext{
(See figure on next page.)

Fig. 3 Osteotomy'defect'site (black arrows) in a rat femur at 5 weeks post-trauma (wpt). A Hematoxylin and eosin (H\&E) stained section of femur in the osteotomy group (OST). The callus of new bone growth (blue arrow) and fibrous connective tissue (CT) (12.5x). B Higher magnification of image A (100x). C Section of femur in polytrauma control (PT-C) group. The callus consists of new bone growth (blue arrow) and immature connective tissue (CT). Note the island of woven bone and bone marrow (red arrow) and focus of inflammation (yellow arrow) (12.5x). D Higher magnification of C (100x). E Higher magnification Figure C (400x). The focus of inflammation consists of multinucleated giant cells (black arrow), macrophages (red arrow), plasma cells (green arrow), lymphocytes (yellow arrow), and hemosiderin-laden macrophages (blue arrow) (chronic hemorrhage). F Section of femur in polytrauma + anti-HMGB1 antibody (PT-Ab HMGB1) group. The majority of the callus consists of new bone growth (blue arrow) with cartilage formation (yellow arrow). G Higher magnification of Figure J (100x). Compare the immature, woven bone (WB) in the callus to the mature, lamellar bone of the femur (LB). Graphical representation of the presence of $\mathbf{H}$ hemosiderin-laden macrophages; I osteoblasts; and $\mathbf{J}$ osteoclasts within the defect at 5 wpt in OST, PT-C, and PT-Ab HMGB1 rats ( $n=4-8 /$ group). The scoring key for hemosiderin-laden macrophages, osteoblast and osteoclasts is mentioned in the material and methods section
} 


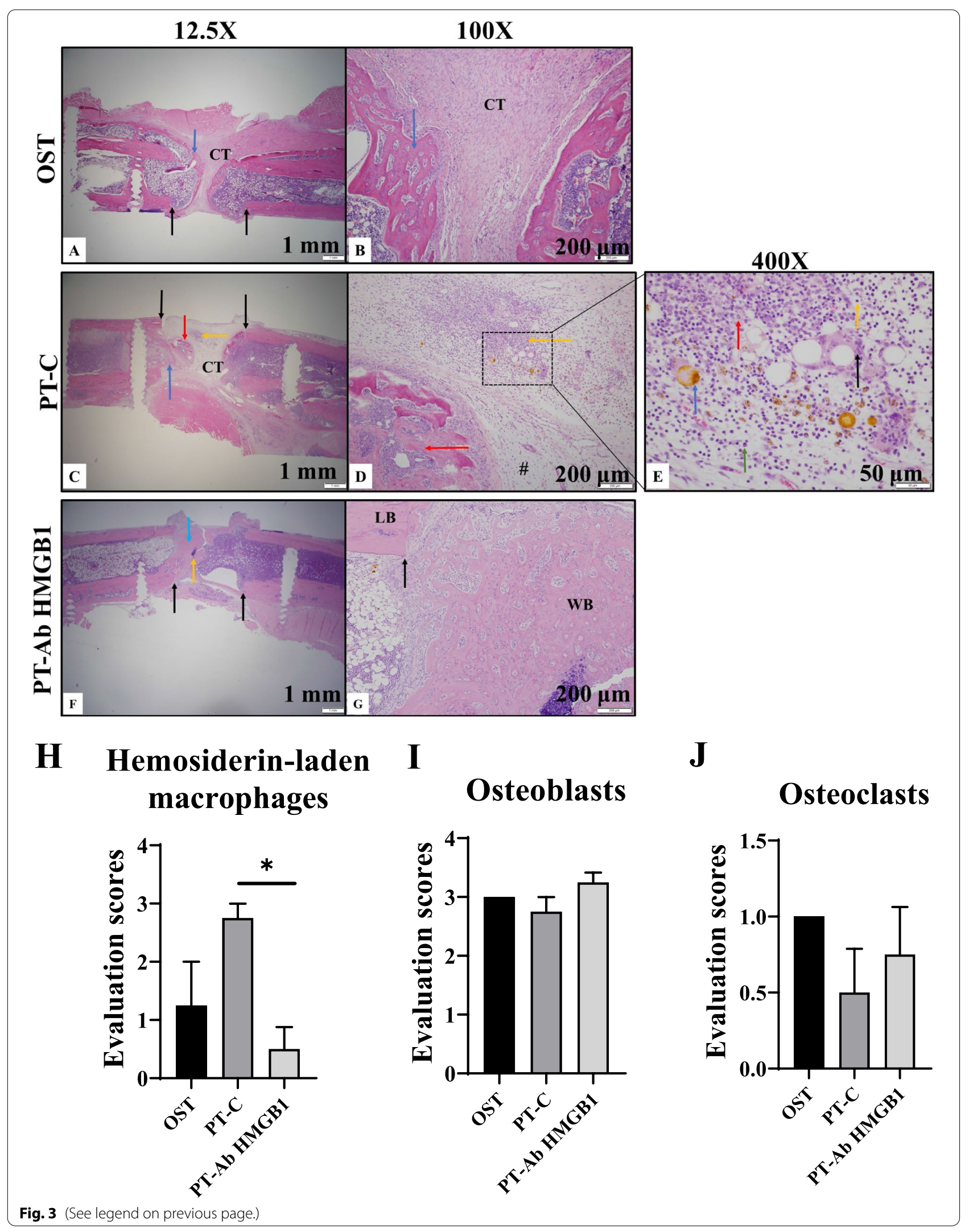




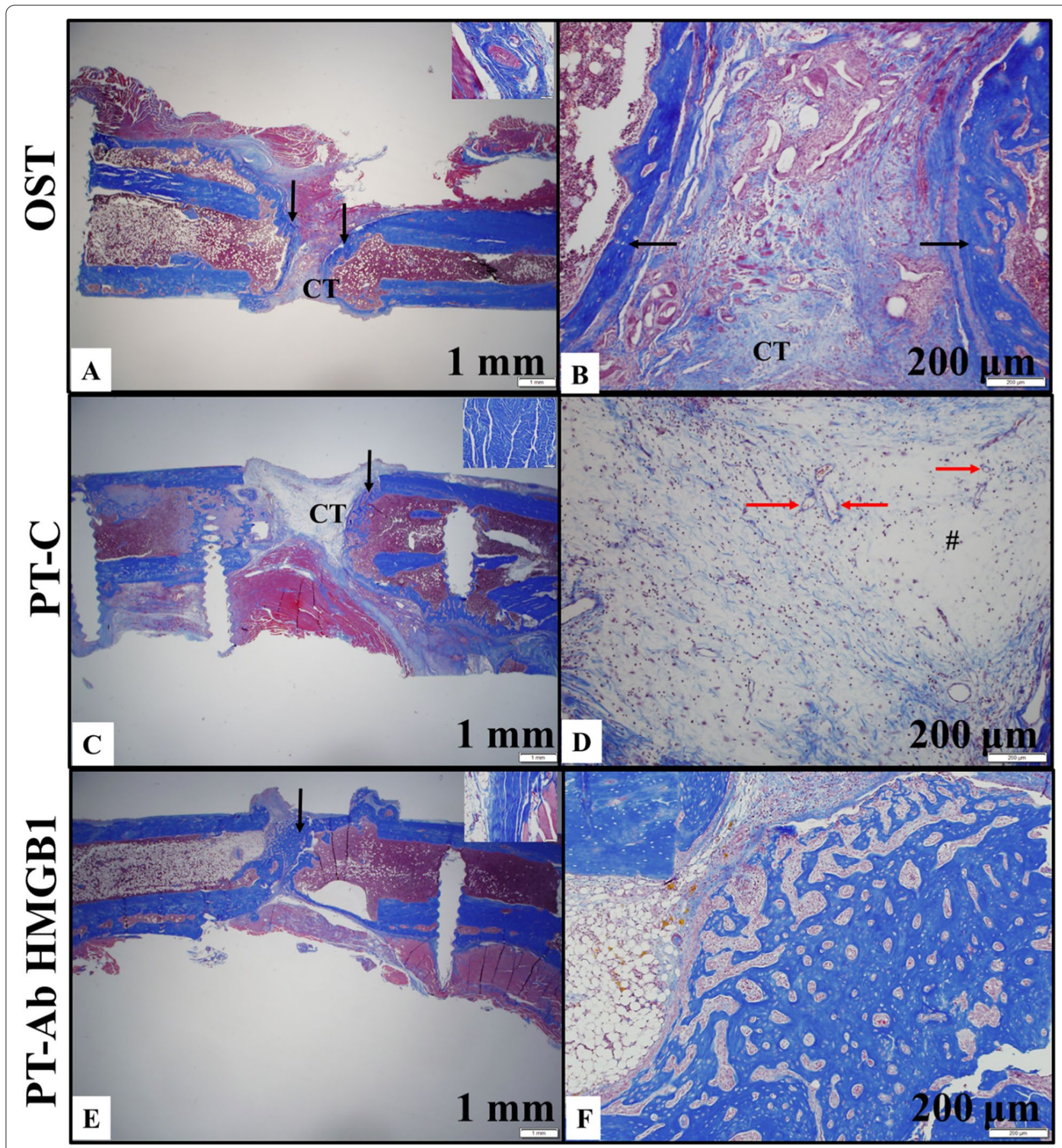

Fig. 4 Decalcified sections of femurs 5 weeks post-trauma (wpt) were stained with Masson's Trichrome (MT) stain. A Section of femur from the osteotomy (OST) group ( $n=4)$. B Higher magnification of image A (100x). C Section of femur from the polytrauma (PT-C) group $(n=4)$. D Higher magnification of Figure C (100x). E Section of rat femur from the polytrauma + Anti-HMGB1 antibody (PT-Ab HMGB1) group $(n=8)$. F Higher magnification of Figure E (100x). Black arrows indicate new bone growth; red arrow indicates new blood vessels; CT - connective tissue

the callus is light blue, with more cells and sparse, disorganized collagen fibers compared to the mature connective tissue, stained deep blue, that has fewer cells, and densely packed organized collagen fibers (Fig. 4D). In
PT-Ab HMGB1 rats the collagen fibers were distinctively denser, with mature connective tissue indicated by deep saturation compared to PT-C whose connective tissue, indicated by light saturation, had more cells and showed 
sparse disorganized collagen fibers. Majority of the callus consists of new bone growth (Fig. 4E and F). There was no qualitative difference between the intensity of staining between immature, woven bone and mature, lamellar bone and the mature connective tissue in a tendon attached to the femur as shown in the inset (Fig. 4E). Collectively, these observations confirm the quality of collagen in the extracellular matrix that determines the form and function of the bone.

\section{HMGB1 promotes $\gamma \delta T C R^{+} T$ cells depletion at the fracture site in PT}

Since PT-Ab HMGB1 rats displayed increased bone formation than PT-C rats, we decided to profile the myeloid and lymphoid cells infiltrating the fracture site of OST, PT-C, PT-IgY, and PT-Ab HMGB1 rats and determine if HMGB1 had a role in modulating a dysregulated immune response at the injury site. At $1 \mathrm{wpt}$, cells from the fracture site were collected and stained for immunophenotyping. On average, we harvested $\sim 3$ million cells from the fracture site. Immunophenotyping analysis of immune cells revealed a slightly increased number of $\mathrm{CD}^{-} \mathrm{CD}^{+} 5^{+}$leukocytes in PT-C rats and PT-IgY rats than OST and PT-Ab HMGB1 at $1 \mathrm{wpt}$, however, not statistically significant (Supplementary Figure S-2A). No differences in macrophages $/ \mathrm{M} \phi$ (i.e., $\mathrm{CD} 3^{-} \mathrm{CD} 45^{+} \mathrm{CD} 68^{+} \mathrm{CD} 86^{+}$(M1) and $\left.\mathrm{CD}^{-} \mathrm{CD} 45^{+} \mathrm{CD} 68^{+} \mathrm{CD} 163^{+}(\mathrm{M} 2)\right)$ occurred between all groups (Supplementary Figure S-2B and C).

Among the lymphoid cells, the $\alpha \beta T$ cells and the $\gamma \delta \mathrm{T}$ cells were quantified in the fracture site at $1 \mathrm{wpt}$ and the cell counts are listed in Table 2. There were no differences in $\mathrm{CD}^{+}{ }^{+} \mathrm{T}$ cell, and $\mathrm{CD}^{+} \mathrm{T}$ cell percent counts across all groups (Fig. 5A-C). The percentage of $\mathrm{CD}^{+} \mathrm{CD}^{+}$ $\mathrm{T}$ cells were slightly increased in PT-C rats and PT-IgY rats than OST and PT-Ab HMGB1 at 1 wpt, however, there was nostatistical significance (Fig. 5D). The percent of $\gamma \delta^{+}$TCR T cells decreased in PT-C $(p=0.01)$ and PT-IgY $(p=0.006)$ compared to OST. Interestingly, the percentage of $\gamma \delta^{+} \mathrm{TCR}$ T cells was increased in the fracture site of PT-Ab HMGB1 compared to PT-C $(p=0.13)$ and PT-IgY ( $p=0.04)$ (Fig. 5E).

\section{Discussion}

This study aimed to establish the role of secreted HMGB1 in mediating immune dysregulation in PT associated with delayed fracture healing. The main findings of this study are that the PT-Ab HMGB1 rats demonstrated increased fracture healing with enhanced bone formation within the fracture defect compared to PT-C rats at $5 \mathrm{wpt}$. Additionally, severe depletion of $\gamma \delta \mathrm{TCR}$ $+\mathrm{T}$ cells counts was observed in PT-C and PT-IgY rats compared to OST. Interestingly, the $\gamma \delta \mathrm{TCR}^{+} \mathrm{T}$ cells counts remained significantly elevated at $1 \mathrm{wpt}$ in $\mathrm{PT}-\mathrm{Ab}$ HMGB1 and OST rats.

In the context of oral bone regeneration, HMGB1 has a beneficial role due to it's ability to initiate an acute inflammatory response to activate regenerative pathways $[9,21]$. While some studies have reported the osteogenic role of HMGB1 in mediating bone regeneration of fractures in single injury models $[16,27,28]$, some other studies have also suggested its detrimental role in impairing tissue regeneration potentiating remote organ injury $[10,29]$. Yet, some others have recently presented the exogenous supplementation of HMGB1 at the fracture site to promote bone regeneration, demonstrating that HMGB1 primes MSCs for osteogenic differentiation [30, 31]. However, the findings from a series of experiments conducted by Lee et al. clarified the confusion about the role of HMGB1 in fracture healing, demonstrating that HMGB1 only in its fully reduced form (fr-HMGB1) has regenerative properties as opposed to its disulfide form (ds-HMGB1) [19]. They further suggest that the posttranslational modifications of HMGB1 determines its role in inflammation and immunity. Further, Lee et al. also demonstrated that complete blocking of HMGB1 with glycyrrhizin resulted in delayed fracture healing [31]. We posit that while secreted HMGB1 is needed for

Table 2 Relative counts of T cells in the fracture site at 1 week post-trauma (wpt) in osteotomy rats (OST), polytrauma rats (PT-C), PT rats with chicken IgY isotype (PT-IgY), and PT rats with anti-HMGB1 (PT-Ab HMGB1) rats. OST $(n=5), \mathrm{PT}-\mathrm{C}(n=5), \mathrm{PT}-\mathrm{lgY}(n=4)$ and PT-Ab HMGB1 $(n=8)$ rats, respectively. ${ }^{*} p<0.05$ for OST vs PT-IgY and PT-C and ** $p<0.05$ for PT-Ab HMGB1 vs PT-IgY and PT-C

\begin{tabular}{|c|c|c|c|c|}
\hline \multirow[t]{2}{*}{ T cells } & \multicolumn{4}{|c|}{ Cell counts (Mean \pm SD) } \\
\hline & OST & PT-C & PT-IgY & PT-Ab HMGB1 \\
\hline $\mathrm{CD}^{+} \mathrm{T}$ cells & $2233 \pm 855$ & $2052 \pm 877$ & $2072 \pm 339$ & $2038 \pm 536$ \\
\hline $\mathrm{CD}^{+}{ }^{+} \mathrm{CD} 4^{+} \mathrm{T}$ cells & $1632 \pm 650$ & $1446 \pm 580$ & $1393 \pm 338$ & $1458 \pm 418$ \\
\hline $\mathrm{CD}^{+} \mathrm{CD}^{+} \mathrm{T}$ cells & $344 \pm 154$ & $331 \pm 224$ & $334 \pm 117$ & $301 \pm 232$ \\
\hline $\mathrm{CD}^{+} \mathrm{CD}^{+}{ }^{+} \mathrm{CD} 8^{+} \mathrm{T}$ cells & $156 \pm 82$ & $208 \pm 104$ & $370 \pm 77$ & $173 \pm 92$ \\
\hline $\mathrm{CD}^{+}{ }^{+} \delta \mathrm{TCR}^{+} \mathrm{T}$ cells & $237 \pm 104^{*}$ & $105 \pm 36$ & $96 \pm 33$ & $234 \pm 57^{* *}$ \\
\hline
\end{tabular}




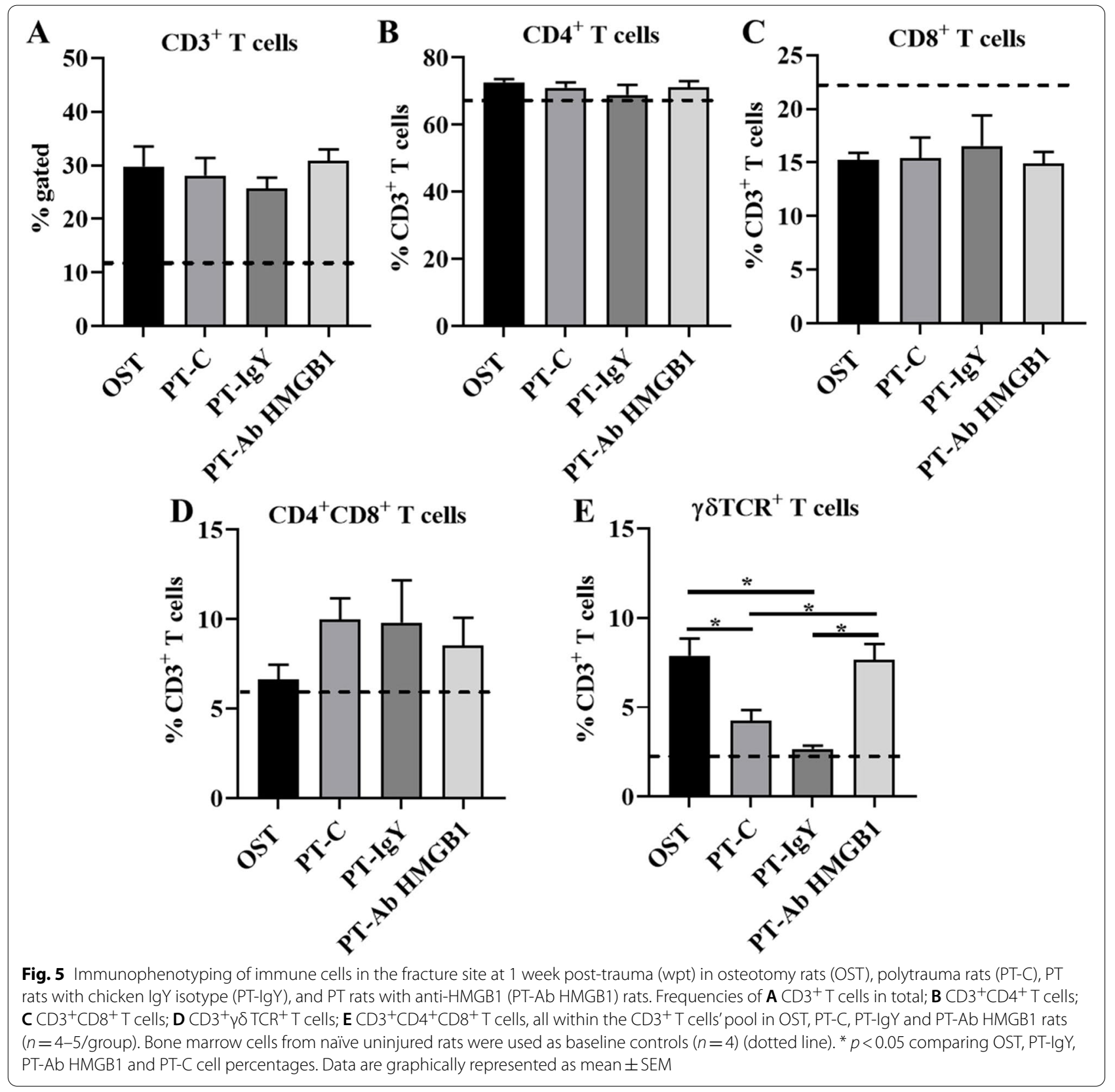

regulating bone regeneration, its regenerative function is primarily concentration-dependent. To our knowledge, this is the first study to assess bone regeneration following neutralization of early-secreted HMGB1 immediately following injuries in a polytrauma rat model.

It is clear structural reconstitution of bone healing after fractures relies on both innate and adaptive immune responses during the entire inflammatory phase. If the immune cell kinetics are disturbed, the essential immune cells required for callus formation become unavailable, thereby delaying or impairing bone healing. As for normal fracture healing, the inflammatory phase begins at the time of injury and lasts up to 5 days, followed by the soft callus formation phase. However, in the event of delayed fracture healing, the inflammatory phase extends to $>5$ dpt reviewed in $[15,32]$. Khassawna et al. demonstrated that $\mathrm{T}$ cells, rather than $\mathrm{B}$ cells, contributed to improved bone extracellular matrix deposition, collagen organization, and fracture healing at 1 wpt [6]. Fractured bones from mice lacking mature $\mathrm{T}$ cells were densely mineralized and stiffer but significantly compromised bone quality compared to the fractured bones from 
mice with normal $\mathrm{T}$ cell counts. This phenotype correlated well with collagen deposition and osteoblast distribution within the fracture site $[6,33]$. Since the impact of $\mathrm{T}$ cells and their subtypes on fracture repair is gaining much attention, and because HMGB1 functions as a central cytokine for all lymphoid cells, we proceeded to capture a snapshot of the $\alpha \beta$ T cells and $\gamma \delta$ T cells present in the fracture space at $1 \mathrm{wpt}$. While the $\mathrm{CD} 4^{+} \mathrm{T}$ cells and $\mathrm{CD}^{+} \mathrm{T}$ cell counts within the $\mathrm{CD}^{+} \mathrm{T}$ cell pool in the fracture space were not comparable between all groups, there was an increasing trend in the $\mathrm{CD} 4^{+} \mathrm{CD} 8^{+}$ (double-positive) T cells in PT-C and PT-IgY rats, but this increase was not statistically significant. The doublepositive T cells express $\alpha \beta T C R s$ and they share the features of both $\mathrm{CD}^{+}$as well as $\mathrm{CD} 8^{+} \mathrm{T}$ cells. They have been previously found enriched in tissues at the site of inflammation in various pathological settings, including cancers and rheumatoid arthritis, [17, 34, 35]. However, $\mathrm{CD} 4^{+} \mathrm{CD} 8^{+}$double-positive $\mathrm{T}$ cells' role is mostly understudied in trauma. Their function remains controversial, with conflicting reports describing cytotoxic and suppressive roles for these cells, and is an ongoing debate. This study is the first report describing $\mathrm{CD} 4{ }^{+} \mathrm{CD} 8^{+}$double-positive $\mathrm{T}$ cells in the long bone fractures. Further research is needed to assess the possible involvement of double-positive $\mathrm{T}$ cells in the fracture site's immune surveillance and bone regeneration in $\mathrm{PT}$.

The $\gamma \delta \mathrm{TCR}^{+} \mathrm{T}$ cells are prevalent at the injury sites and are known for their immune surveillance role with a final goal to enhance healing [20]. Colburn et al. have reported a particular pattern in the $\gamma \delta \mathrm{TCR}^{+} \mathrm{T}$ cell dynamics at the fracture site that delays the healing cascade, a phenotype possibly occurring due to the heavy oxidative burst that prevails at the injury site [19]. Other investigations emphasized the importance of the $\gamma \delta \mathrm{TCR}^{+} \mathrm{T}$ cells in bone regeneration, demonstrating a strong potential of these cells to serve as precursors of possible adverse immune effects of immunosuppressive therapies [36, 37]. Further, in vitro investigations demonstrated that upon activation, the $\gamma \delta \mathrm{TCR}^{+} \mathrm{T}$ cells tend to inhibit osteoclastogenesis and promote osteoblastogenesis via the secretion of specific growth factors and cytokines [38]. We infer from our findings that despite the inconsequential role of $\mathrm{CD}^{+}$and $\mathrm{CD} 8^{+} \mathrm{T}$ cells in polytraumatic fractures, the innate-lymphocytes, particularly the $\gamma \delta \mathrm{TCR}$ ${ }^{+} \mathrm{T}$ cell, may have a potential role during fracture healing. These findings are as per the previous reports demonstrating that it is not the $\alpha \beta T$ cells, but rather, the $\gamma \delta \mathrm{T}$ cells are essential players of ossification and bone regeneration following fractures [36]. However, fundamental information about the biological link between the $\gamma \delta \mathrm{TCR}$ $+\mathrm{T}$ cells and HMGB1 in the context of polytraumatic fracture healing needs further elucidation.
The limitation of this study is that it represents a snapshot of the immune cell profile at $1 \mathrm{wpt}$, which may change dynamically over time. This time-point was chosen because of the prolonged inflammatory phase in delayed fracture healing, and immune cell infiltration that was detected at 1 week in previous fracture repair studies [6]. Evaluating multiple time points earlier and later than 1 week would explain whether the magnitude and phenotypic distribution of $\gamma \delta \mathrm{TCR}^{+} \mathrm{T}$ cell dynamics evolves temporally at the fracture site after multiple concurrent injuries. Additionally, since there was no difference in cellular distributions in the PT-IgY group compared to PT-C, bone healing was evaluated using the PT-C group as the control group. Finally, toward the ultimate goal of therapeutic immunomodulation, follow-up studies should employ gain- and loss-of-function techniques to elucidate the functions of $\gamma \delta \mathrm{TCR}^{+} \mathrm{T}$ cells with and without neutralizing extracellular HMGB1 in PT.

\section{Conclusion}

In concordance with the previously published reports on $\mathrm{T}$ cells in fracture healing, we showed that PT did not significantly influence $\alpha \beta$ T cell frequencies; instead, it decreased $\gamma \delta \mathrm{TCR}^{+} \mathrm{T}$ cell frequencies within the fracture space. Following neutralization of secreted HMGB1 post-polytraumatic injuries in rats, we established a role for HMGB1 in bone regeneration. We conclude that by regulating extracellular HMGB1 levels, the bone formation process was guided to allow for proper ossification leading to improved bone quality and resolution accompanied by an increase in $\gamma \delta \mathrm{TCR}^{+} \mathrm{T}$ cells at the fracture site. Moving forward, by selectively targeting danger molecules such as HMGB1 either locally or systemically with potential therapeutic biologics, new interventions may determine efficient regulation of deranged immune responses to promote fracture repair in PT patients.

\section{Supplementary Information}

The online version contains supplementary material available at https://doi. org/10.1186/s40634-022-00453-3.

Additional file 1. Micro-computed tomography $(\mu \mathrm{CT})$ analysis results of bone regeneration in polytrauma (PT) rats following treatment with three doses (1dose/day 0, 1 and 2) of anti-HMGB1 antibody (PT-HMGB1 3x).

Additional file 2. HMGB1 increases infiltration of myeloid cells at the fracture site in polytrauma (PT).

\section{Acknowledgments}

We thank Ms. Christina Sandoval and Mr. Kinton Armmer for animal surgery, and technical support. We thank MAJ Nathan Wienandt, DVM, a boardcertified veterinary pathologist, for his assistance with providing histological scores, imaging and interpretation of histological results. We also thank $\mathrm{Mr}$. Scott Kelly, Mr. Pham Phong, Dr. Lauren Mangum and Ms. Desiree Romano for animal surgery, assistance, sample collection and laboratory support. We thank Dr. Stefanie Shiels for reviewing the article and providing her valuable suggestions. 


\section{Author's contributions}

JCW conceived this study, acquired funding and assisted with the interpretation of results. JJA performed the 5 weeks study endpoint to evaluate bone healing with oversight from JCW. ALL analyzed the $\mu \mathrm{CT}$ data for bone healing. PJM performed the 1 week study endpoint to evaluate immune cell dynamics with the oversight from JCW. ALL and PJM collected samples and performed experiments. PJM analyzed data, interpreted results, prepared figures, and drafted the manuscript. All authors revised, edited and approved the final version of the manuscript.

\section{Funding}

This work was funded and supported by the Combat Casualty Care Research Program (CCCRP)

\section{Availability of data and materials}

The datasets generated during and/or analyzed during the current study are available from the corresponding authors on reasonable request.

\section{Declarations}

Ethics approval and consent to participate Not applicable.

\section{Consent for publication}

Not applicable.

\section{Competing interests}

The authors declare no competing interests.

Received: 10 September 2021 Accepted: 3 February 2022

Published online: 28 February 2022

\section{References}

1. Mills LA, Aitken SA, Simpson AHR (2017) The risk of non-union per fracture: current myths and revised figures from a population of over 4 million adults. Acta Orthop 88(4):434-439

2. $\mathrm{Xu} J$ et al (2016) IL-12p40 impairs mesenchymal stem cell-mediated bone regeneration via CD4+ T cells. Cell Death Differ 23(12):1941-1951

3. Störmann P et al (2020) Monotrauma is associated with enhanced remote inflammatory response and organ damage, while polytrauma intensifies both in porcine trauma model. Eur J Trauma Emerg Surg 46(1):31-42

4. Bidwell JP, Yang J, Robling AG (2008) Is HMGB1 an osteocyte alarmin? J Cell Biochem 103(6):1671-1680

5. Rapp AE et al (2016) Fracture Healing Is Delayed in Immunodeficient NOD/ scid- IL2Ry cnull Mice. PLoS One 11(2):e0147465

6. El Khassawna T et al (2017) T lymphocytes influence the mineralization process of bone. Front Immunol 8:562

7. Horst K et al (2015) Local inflammation in fracture hematoma: results from a combined trauma model in pigs. Mediators of inflammation 2015:126060

8. Davis HM et al (2019) High mobility group box 1 protein regulates osteoclastogenesis through direct actions on osteocytes and osteoclasts in vitro. J Cell Biochem 120(10):16741-16749

9. Aoyagi $\mathrm{H}$ et al (2018) HMGB1-induced inflammatory response promotes bone healing in murine tooth extraction socket. J Cell Biochem 119(7):5481-5490

10. Braun CK et al (2017) Early structural changes of the heart after experimental polytrauma and hemorrhagic shock. PloS one 12(10):e0187327

11. Tzioupis C, Giannoudis PV (2007) Prevalence of long-bone non-unions. Injury 38:53-59

12. Tsung A et al (2005) The nuclear factor HMGB1 mediates hepatic injury after murine liver ischemia-reperfusion. J Exp Med 201(7):1135-1143

13. Serra A et al (2012) Lymphocytes control bone fracture healing by programming the mineralisation capacity of migratory osteogenic precursors. Ann Rheum Dis 71(Suppl 1):A63-A63

14. Sakamoto Y et al (2020) High-mobility group box 1 induces bone destruction associated with advanced oral squamous cancer via RAGE and TLR4. Biochem Biophys Res Commun 531(3):422-430
15. Claes L, Recknagel S, Ignatius A (2012) Fracture healing under healthy and inflammatory conditions. Nat Rev Rheumatol 8(3):133-143

16. Santo AlE, et al (2014) The role of alarmins in fracture repair. in European Calcified Tissue Society Congress 2014. BioScientifica. https://doi.org/10.1530/ boneabs.3.OC 1.5

17. De Maria A et al (1987) CD3+4-8-WT31-(T cell receptor $\gamma+$ ) cells and other unusual phenotypes are frequently detected among spontaneously interleukin 2-responsive T lymphocytes present in the joint fluid in juvenile rheumatoid arthritis. A clonal analysis. Eur J Immuno 17(12):1815-1819

18. Robinson AP et al (2013) High-mobility group box 1 protein (HMGB1) neutralization ameliorates experimental autoimmune encephalomyelitis. J Autoimmun 43:32-43

19. Colburn NT et al (2009) A role for $\gamma / \delta T$ cells in a mouse model of fracture healing. Arthritis \& Rheumatism: Official Journal of the American College of Rheumatology 60(6):1694-1703

20. Ribot JC, Lopes N, Silva-Santos B (2020) $\gamma \delta$ t cells in tissue physiology and surveillance. Nat Rev Immunol 21(4):221-232

21. Yoshihara-Hirata C et al (2018) Anti-HMGB1 neutralizing antibody attenuatesperiodontal inflammation and bone resorption in a murine periodontitis model. Infect Immun 86(5):e00111-18

22. Percie du Sert N et al (2020) The ARRIVE guidelines 2.0: Updated guidelinesfor reporting animal research. Br J Pharmacol 40(9):1769-1777

23. Mangum LH et al (2019) Burn and thoracic trauma alters fracture healing, systemic inflammation, and leukocyte kinetics in a rat model of polytrauma. J Orthop Surg Res 14(1):58

24. Otsu N (1979) A threshold selection method from gray-level histograms. IEEE Trans Syst Man Cybern 9(1):62-66

25. Boes KM, Durham AC (2017) Bone marrow, blood cells, and the lymphoid/ lymphatic system. Pathologic basis of veterinary disease 2017:742-804

26. Türkmen $\mathrm{N}$ et al (2008) The significance of hemosiderin deposition in the lungs and organs of the mononucleated macrophage resorption system in infants and children. J Korean Med Sci 23(6):1020-1026

27. Chen MQ, Luan JJ (2019) HMGB1 promotes bone fracture healing through activation of ERK signaling pathway in a rat tibial fracture model. Kaohsiung J Med Sci 35(9):550-558

28. Li G, Liang X, Lotze MT (2013) HMGB1: the central cytokine for all lymphoid cells. Front Immunol 4:68

29. Oyama M et al (2020) High-mobility group box 1 released by traumatic occlusion accelerates bone resorption in the root furcation area in mice. J Periodontal Res 56(1):186-194

30. Xue D et al (2017) Local delivery of HMGB1 in gelatin sponge scaffolds combined with mesenchymal stem cell sheets to accelerate fracture healing. Oncotarget 8(26):42098

31. Lee $\mathrm{G}$ et al (2018) Fully reduced HMGB1 accelerates the regeneration of multiple tissues by transitioning stem cells to GAlert. Proc Natl Acad Sci 115(19):E4463-E4472

32. Muire PJ, Mangum LH, Wenke JC (2020) Time course of immune response and immunomodulation during normal and delayed healing of musculoskeletal wounds. Front Immunol 11:1056

33. Toben D et al (2011) Fracture healing is accelerated in the absence of the adaptive immune system. J Bone Miner Res 26(1):113-124

34. Quandt D et al (2014) Peripheral CD4CD8 double positive T cells with a distinct helper cytokine profile are increased in rheumatoid arthritis. PloS one 9(3):e93293

35. Parel Y, Chizzolini C (2004) CD4+ CD8+ double positive (DP) T cells in health and disease. Autoimmun Rev 3(3):215-220

36. Ono T et al (2016) IL-17-producing $\gamma \delta T$ cells enhance bone regeneration. Nat Commun 7(1):1-9

37. Kalyan S (2016) It may seem inflammatory, but some T cells are innately healing to the bone. J Bone Miner Res 31(11):1997-2000

38. Phalke SP, Chiplunkar SV (2015) Activation status of $\gamma \delta$ T cells dictates their effect on osteoclast generation and bone resorption. Bone reports 3:95-103

\section{Publisher's Note}

Springer Nature remains neutral with regard to jurisdictional claims in published maps and institutional affiliations. 